\title{
Effectiveness of Clinical Pharmacist Directed-Diabetes Management
}

\author{
Neha Farheen Tabassum1, N. Himanandini', Manaal Fathima1, M. Sankeerthna1, \\ Nikhil Kumar Vanjari2*, Siva Subrahmanyam Bandaru ${ }^{3}$ \\ ${ }^{1}$ Vaageswari College of Pharmacy, Karimnagar, Telangana, India \\ ${ }^{2}$ Department of Pharmacy Practice, Vaageswari College of Pharmacy, Karimnagar, Telangana, India \\ ${ }^{3}$ Sri Bhadrakali Diabetes Care Centre, Warangal, Telangana, India \\ Email: *nikhil71311@gmail.com
}

How to cite this paper: Tabassum, N.F., Himanandini, N., Fathima, M., Sankeerthna, M., Vanjari, N.K. and Bandaru, S.S. (2019) Effectiveness of Clinical Pharmacist Directed-Diabetes Management. Journal of Diabetes Mellitus, 9, 137-145. https://doi.org/10.4236/jdm.2019.93013

Received: May 11, 2019

Accepted: August 19, 2019

Published: August 22, 2019

Copyright $\odot 2019$ by author(s) and Scientific Research Publishing Inc. This work is licensed under the Creative Commons Attribution International License (CC BY 4.0).

http://creativecommons.org/licenses/by/4.0/

\begin{abstract}
Objective: The main aim of the study is predominately utilizing clinical pharmacist in the provision of continuing diabetic education programs to emphasize and re-emphasize the importance of risk factors, prevention, adherence to medication and behavioral changes to prevent recurrences of the disease, their progression, and ultimately minimize hospitalization. Specific goals are to improve clinical outcomes for patients with diabetes-to maintain optimal plasma glucose concentrations Fasting, Post-prandial Plasma Glucose, and Glycated Hemoglobin. Study design: A prospective interventional study in the Outpatient General Medicine Department, for a period of six months from October 2018 to March 2019. Results: HbAlc levels were reduced from baseline by $-1.107 \pm 0.8634$, Fasting Plasma Glucose levels and Postprandial blood glucose levels were reduced from baseline by $-24.2218 \pm$ 5.70352 and $-30.1891 \pm 1.40592$ respectively. Conclusion: A trained clinical pharmacist by providing diabetes education and care can significantly reduce hyperglycemia, thereby improving the quality of life in diabetes patients and ultimately reducing health care costs associated with these morbidities.
\end{abstract}

\section{Keywords}

Diabetes Mellitus, Clinical Pharmacist, Glycated Hemoglobin, Fasting Plasma Glucose, Post-Prandial Blood Glucose, Diabetes Care and Management

\section{Introduction}

Diabetes mellitus is a chronic progressive metabolic disorder characterized by hyperglycemia with impaired metabolism of carbohydrate, protein, and fat re- 
sulting from absolute Type 1 Diabetes Mellitus or relative Type 2 Diabetes Mellitus deficiency of insulin or both. Diabetes has leaped from 108 million in 1980 to 422 million patients in 2014 . From $4.7 \%$ in 1980 , the global incidence in the ages above 18 years has uprisen to $8.5 \%$ in 2014 [1]. Hyperglycaemia over a long period of time can lead to metabolic disturbances and virtually affects every system of the human body particularly the blood vessels and nerves leading to diabetes mellitus associated macrovascular \& microvascular complications, diabetic foot ulcer and recurrent infections [2]. The essential element in the management of diabetes mellitus includes therapeutic management, self-care management, patient adherence to the prescribed medication and lifestyle modifications. Though the diabetic patients are managed by the physicians, clinical inertia and limited time with patients hamper efforts to meet treatment goals. So, to have an effective delivery in achieving the goal of therapy, it requires a partnership between people and healthcare providers. With the intimate understanding of how medications work and patient care, clinical pharmacist brings a unique and valuable perspective to diabetes care even as the population of patients with diabetes continues to grow [3]. As a vital members of the healthcare team, pharmacist has a significant impact on diabetes care and education by screening patients at high risk for diabetes, setting and monitoring diabetic treatment goals, assessing patient's health status, home glucose meter training, performed physical assessment of patient's feet, skin, blood pressure and weight, blood pressure \& lipid management education and adherence to standards of care [4]. Alterations in behavior to ameliorate plasma glucose levels were observed when interventions were made by a healthcare provider in diabetic patients [5] [6] and adequate intake of fat, protein, and carbohydrate controls metabolism which minimizes the hospitalizations and deaths owing to diabetes [7].

\section{Aim and Objectives}

The main aim of the study is predominately utilizing clinical pharmacist in the provision of Continuous education programs and counseling helps Diabetic patients to emphasize and re-emphasize the importance of risk factors, prevention, adherence to medication and behavioral changes to prevent recurrences of the disease, their progression, and ultimately minimize hospitalization. Specific goals are to improve clinical outcomes for patients with diabetes-to maintain optimal plasma glucose concentrations Fasting, Post-prandial Plasma Glucose, and Glycated Hemoglobin.

\section{Materials and Methods}

Patients were enrolled in the study if they were with type 2 diabetes mellitus who were treated with either oral agents or insulin and had an HbA1C concentration $>7 \%$. Patients are referred to the program by the primary care physician for various indications, such as newly diagnosed diabetes, poor glycemic control, poor adherence, or a combination of these. In addition, the patient was included 
based on an $\mathrm{HbA1C}$ concentration $>7 \%$ and the primary care provider's overall judgment that the patient required additional time at follow-up visits for intensive patient education and monitoring, once enrolled in the study, Each visit is individualized based on patient-specific needs; patients were scheduled to meet with the pharmacist every 4 -weeks for approximately 30 minutes. Patients were followed in the clinic for at least 3 - 4 months. Follow-up is conducted through a combination of telephone appointments and clinic visits. All pharmacists managing patients in this study are clinically trained under Diabetic Educator, bilingual and had spent at least one clinical rotation (6 months) during their Pharm.D course in the Hospital. During clinic appointments, the pharmacist provided diabetes education, medication counseling, monitoring, and management.

\section{Study Design}

A prospective interventional study was carried out in the Sri Bhadrakali Diabetes Clinic, Warangal, Telangana, India. The study was carried out for a period of Six months from October 2018 to March 2019.

Patients with diabetes mellitus with or without co-morbidities visiting the outpatient general medicine department were included in the study. Pregnant women, mentally challenged patients, and critically ill patients were excluded from the study. Patient's demographic details including past medical and medication history, current drug treatment and laboratory values were collected in the data sheet designed as per the need of the study. Educational materials (validated patient information leaflets) and medication counseling were given to the interventional group by the study pharmacist during various follow-up visits.

\section{Statistical Analysis}

Data is collected using a self-designed collection form and analyzed using GraphPad Prism 8. Demographic data values are presented in mean with standard deviation. A confidence level of $99 \%$ is employed and the test results are statistically significant if the P-value is $<0.001$. A One-way ANOVA of repeated measures and Student's paired t-test were applied to Fasting/Post-prandial plasma glucose and Glycated hemoglobin respectively.

\section{Results}

Clinical characteristics: Of the initial 608 Type 2 Diabetic patients who were given Diabetic Education based on patient-specific needs., 401 patients were lost for follow-up and 207 patients were followed up for HbAlc, Fasting Plasma Glucose, and Postprandial Plasma Glucose. A correlation test was also performed to evaluate the relationship between Fasting plasma glucose and Glycated Hemoglobin, both at baseline and 12-weeks (Table 1) (Figure 1) (Figure 2).

$47 \%$ were males and $53 \%$ were females from a total of 207 diabetic patients (Table 2). The mean age was $48.99091 \pm 12.27377$ and $52.94845 \pm 9.790982$ in 
Table 1. Correlation of HbA1c \& Fasting Plasma Glucose.

\begin{tabular}{ccc}
\hline Characterstic & Correlation Coeficent & Interpretation \\
\hline Baseline FPG \& HbA1c & $\mathrm{r}=0.4372$ & Moderate Correlation \\
12-Week FPG \& HbA1c & $\mathrm{r}=0.2431$ & Low Correlation \\
\hline
\end{tabular}

Table 2. Age and Gender Distribution.

\begin{tabular}{cc}
\hline Sample Size & $\mathrm{n}=207$ \\
\hline Gender & Males: $47 \%$ \\
& Females: $53 \%$ \\
Age in years & Males: $48.99091 \pm 12.27377$ \\
& Females: $52.94845 \pm 9.790982$ \\
\hline
\end{tabular}

Correlation of Baseline HbA1c \& FPG

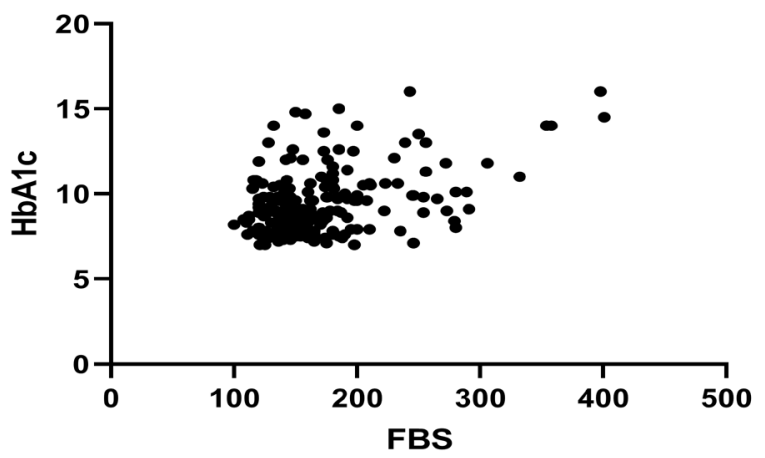

Figure 1. Correlation of baseline HbAlc (\%) \& fasting plasma glucose (mg/dl).

Correlation of 12-week HbA1c \& FPG

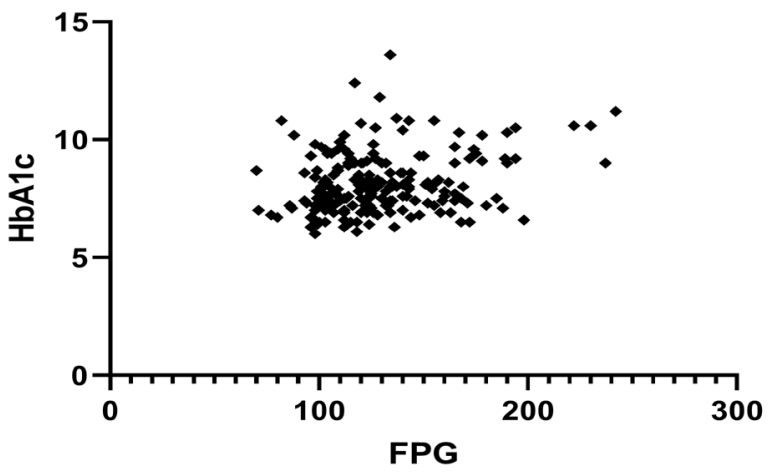

Figure 2. Correlation of 12-week glycated HbA1c (\%) \& fasting plasma glucose (mg/dl).

males and females respectively. Changes in Glycemic control from baseline to 12-weeks is represented in tabulated form (Table 3). The mean HbAlc levels at the beginning of the study were $9.417341 \pm 1.821157$. At the end of the study, HbAlc levels were reduced from baseline by $-1.107 \pm 0.8634$ with a $\mathrm{P}$ value $<$ 0.0001 (Figure 3). The mean Fasting Plasma Glucose levels at the beginning of the study was $173.072 \pm 61.49741$. At the end of the study, Fasting Plasma Glucose 
Table 3. Changes in Glycemic control from baseline to 12-weeks.

\begin{tabular}{cccccc}
\hline Characteristics & $\begin{array}{c}\text { Baseline } \\
(\text { Mean } \pm \text { SD })\end{array}$ & $\begin{array}{c}8 \text { Weeks } \\
(\text { Mean } \pm \text { SD })\end{array}$ & $\begin{array}{c}12 \text { Weeks } \\
(\text { Mean } \pm \text { SD })\end{array}$ & $\begin{array}{c}\text { Changes from Baseline } \\
\text { Mean difference } \pm \text { SD }\end{array}$ & $\begin{array}{c}\text { P value } \\
\text { HbA1C\% }\end{array}$ \\
\hline $9.417341 \pm 1.821157$ & - & $8.44372 \pm 1.406647$ & $-1.107 \pm 0.8634$ & $<0.0001$ \\
Fasting plasma glucose $(\mathrm{mg} / \mathrm{dl})$ & $173.072 \pm 61.49741$ & $162.9614 \pm 61.15363$ & $148.8502 \pm 55.79389$ & $-24.2218 \pm 5.70352$ & $<0.0001$ \\
Postprandial plasma glucose $(\mathrm{mg} / \mathrm{dl})$ & $245.2222 \pm 74.15605$ & $230.8357 \pm 73.15527$ & $215.0331 \pm 72.75013$ & $-30.1891 \pm 1.40592$ & $<0.0001$ \\
\hline
\end{tabular}

\section{Changes in Glycated Hemoglobin}

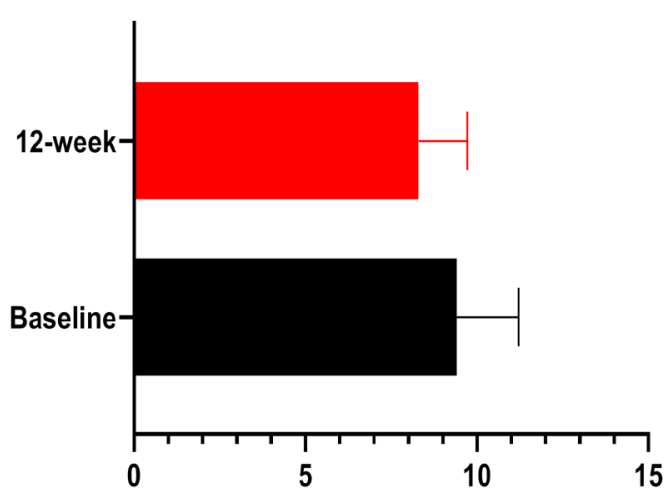

Figure 3. Changes in glycated hemoglobin (\%).

levels were reduced from baseline by $-24.2218 \pm 5.70352$ with a $\mathrm{P}$ value $<0.0001$ (Figure 4). The mean Fasting Plasma Glucose levels at the beginning of the study were $173.072 \pm 61.49741$. At the end of the study, Fasting Plasma Glucose levels were reduced from baseline by-30.1891 \pm 1.40592 with a $\mathrm{P}$ value $<0.0001$ (Figure 5).

\section{Discussion}

Several studies have acknowledged the importance of pharmacist provided counseling in diabetes patients. In the present study, 207 patients were followed up for HbA1c, Fasting Plasma Glucose and Postprandial Plasma Glucose. At the end of the study, $\mathrm{HbAlc}$ levels were reduced from baseline by $-1.107 \pm 0.8634$ with a $\mathrm{P}$ value $<0.0001$, Fasting Plasma Glucose levels were reduced by -24.2218 \pm 5.70352 with a $\mathrm{P}$ value $<0.0001$, Postprandial Plasma Glucose levels were reduced by $-30.1891 \pm 1.40592$ with a $P$ value $<0.0001$. Clinically significant improvements are reported.

The efficacy of Diabetic Pharmaceutical care was conducted for a period of 12 months by the Fremantle Study. Patients were counseled for lifestyle changes, use of daily medication, Dietary pattern and were assessed at baseline, 6 months and 12 months. The main outcome measure was changed in HbA1C. The study concluded that the 12-month PC program in type 2 diabetes reduced glycemia and blood pressure. Pharmacist involvement contributed to improvement in HbAlc independently of pharmacotherapeutic changes [8]. Cioffi et al. conducted the study to determine the effect of a clinical pharmacist directed diabetes 


\section{Baseline to 12-week Fasting Plasma Glucose}

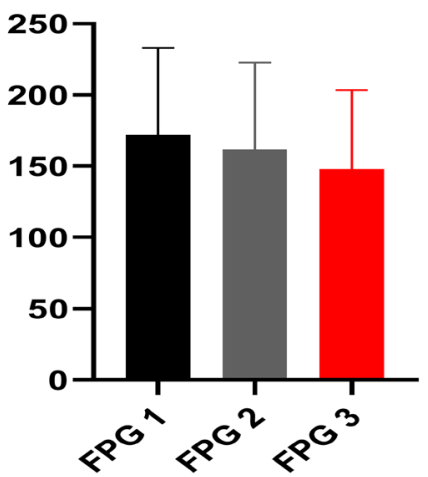

Figure 4. Baseline to 12-week fasting plasma glucose (mg/dl).

\section{Baseline to 12-week PostPrandial Plasma Glucose}

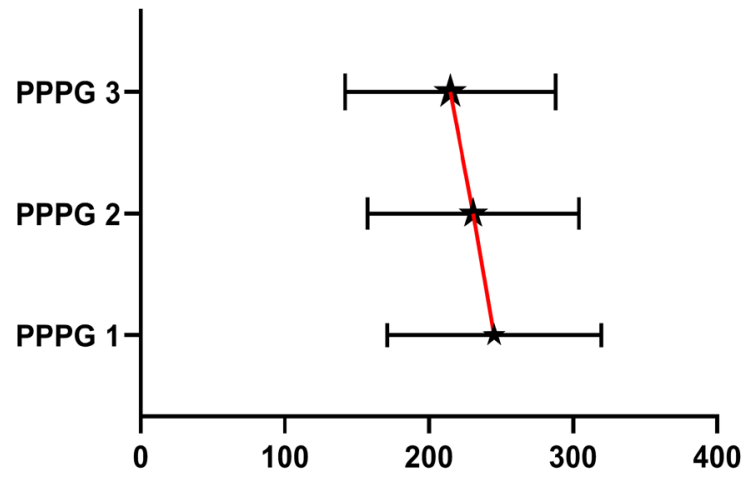

Figure 5. Baseline to 12 weeks postprandial plasma glucose $(\mathrm{mg} / \mathrm{dl})$.

management clinic on glycemic control and cardiovascular and renal parameters in patients with type 2 diabetes. The primary endpoint was the impact of $9-12$ months of participation in the clinic on HbA1C. The study demonstrated that a clinical pharmacist can effectively care for patients with diabetes referred by their primary care provider because of poor glycemic control [9]. Gerber et al. conducted a study to assess the impact on healthcare utilization and costs of pharmacist consultations provided to patients with diabetes. The study suggested that pharmacist consultations provided to patients with diabetes can decrease total healthcare costs in a health maintenance organization [10]. Cranor et al., assessed 5 years outcome after the initiation of community-based pharmaceutical care services for diabetic patients. A Quasi-experimental, cohort study, longitudinal pre-post was conducted in twelve community pharmacies in Asheville, N.C. The study concluded that patients with diabetes who received ongoing pharmaceutical care services maintained improvement in $\mathrm{HbAlc}$ over time, and employers experienced a decline in mean total direct medical costs [11]. The impact of a specially designed education program, diabetes-related knowledge, and compliance of insulin-dependent diabetic patients were investigated by Powell et al. The program was successful in producing improvements in 
both compliance and knowledge but a need for individualization of patient education efforts was indicated [12]. Odegard et al. studied the effect of intervention by a pharmacist on improving glycemic control; secondary endpoints were medication appropriateness and self-reported adherence. Seventy-seven subjects, were randomized to receive a pharmacist intervention $(n=43)$ or usual care $(n$ = 34) for 6 months, followed by a 6 -month usual-care observation period for both groups. The study concluded that pharmacist intervention did allow for similar HbA1C control with fewer physician visits but did not significantly improve glycemic control. Medication appropriateness and self-reported adherence compared with usual care in individuals with poorly controlled diabetes were not changed [13]. Kiel and McCord evaluated the changes in clinical outcomes for patients enrolled in a pharmacist-coordinated diabetes management program. Data collection included baseline and follow-up values for $\mathrm{HbA1C}$ and lipids as well as the frequency of adherence to preventive care, including annual foot and eye examinations and daily aspirin therapy. The study concluded that for enrolled patients, pharmacist-coordinated diabetes supervision was effective in improving clinical laboratory tests. Significant improvements were observed in $\mathrm{HbA1C}$ and LDL values as well as the frequency of adherence to preventive care [14].

\section{Conclusions}

The observed results might find new insights into diabetes management differently by using clinical pharmacists rather than focusing in addition to next line therapy of the hypoglycemic agents. Further acceptance of efforts of clinical pharmacists by diabetic patients may lead to the establishment of Diabetic care centers in developing countries, which leads to improvement of glycemic control, decreased rate of complications, decreased economic burden, hospitalizations and deaths. Over and above that, setting up federal mechanisms to prevent risk factors for diabetes, evaluating key hurdles in identification, management, glycemic control, diabetes prevention from primary care level and aid in the collection, analyzing of diabetic patient data on a periodic basis could promote the health and well-being of the nation.

In our study, we conclude that a trained clinical pharmacist by providing diabetes education and care can significantly reduce hyperglycemia, thereby improving the quality of life in diabetes patients. Pharmacists are in a major position to make certain that the use of medications is appropriate and safe. Patient counseling on medication, dietary pattern, physical exercises, regular monitoring of plasma glucose and annual, semi-annual Diabetes, cardiovascular and other body systems functioning tests could keep diabetic patients healthy. Providing general diabetes education, designing individual dietary instructions, regular laboratory monitoring parameters exercise decrease microvascular and macrovascular complications and ultimately reduce health care costs associated with these morbidities. To be an effective counselor, the pharmacist should up- 
date his knowledge regarding the latest developments and should possess adequate verbal and non-verbal communication skills. It is important that pharmacists' document evidence shows positive outcomes in the management of patients with diabetes. Dissemination of this information would be valuable because it could raise the awareness of other healthcare professionals regarding pharmacists as effective clinicians in the care of patients with diabetes. This increased awareness could elevate the profile of pharmacists. Limitations of the study include a lack of control group and the small number of patients, further studies focusing on pharmacist's efficacy in diabetic complications rather than glycemic control alone are valued.

\section{Conflicts of Interest}

The authors declare no conflicts of interest regarding the publication of this paper.

\section{References}

[1] The Emerging Risk Factors Collaboration (2010) Diabetes Mellitus, Fasting Blood Glucose Concentration, and Risk of Vascular Disease: A Collaborative Meta-Analysis of 102 Prospective Studies. The Lancet, 375, 2215-2222. https://doi.org/10.1016/S0140-6736(10)60484-9

[2] Mohan, V., Sandeep, S., Deepa, R., Shah, B. and Varghese, C. (2007) Epidemiology of Type 2 Diabetes: Indian Scenario. Indian Journal of Medical Research, 125, 217-230.

[3] Misita, C.P. (2013) Clinical Pharmacists in Outpatient Diabetes Care: Essential Members of the Multi Disciplinary Team. Clinical Diabetes, 31, 162-165. https://doi.org/10.2337/diaclin.31.4.162

[4] Palain, S., Chhetri, A., Prabhu, M., Rajan, S. and Shankar, P. (2004) Role of Pharmacist in Counseling Diabetes Patients. The Internet Journal of Pharmacology, 4.

[5] Johnson, J.A. (1996) Self-Efficacy Theory as a Framework for a Community Pharmacy-Based Diabetes Education Programs. The Diabetes Educator, 22, 237-241. https://doi.org/10.1177/014572179602200307

[6] Norris, S.L., Lau, J., Smith, S.J., et al. (2002) Self-Management Education for Adults with Type 2 Diabetes: A Meta-Analysis of the Effect on Glycemic Control. Diabetes Care, 25, 1159-1171. https://doi.org/10.2337/diacare.25.7.1159

[7] The Diabetes Control and Complications Trial Research Group (1993) The Effect of Intensive Treatment of Diabetes on the Development and Progression of Long-Term Complications in Insulin-Dependent Diabetes Mellitus. The New England Journal of Medicine, 329, 977-986. https://doi.org/10.1056/NEJM199309303291401

[8] Clifford, R.M., Davis, W.A., Batty, K.T. and Davis, T.M. (2005) Effect of a Pharmaceutical Care Program on Vascular Risk Factors in Type 2 Diabetes: The Fremantle Diabetes Study. Diabetes Care, 28, 771-776. https://doi.org/10.2337/diacare.28.4.771

[9] Cioffi, S.T., Caron, M.F., Kalus, J.S., Hill, P. and Buckley, T.E. (2004) Glycosylated Hemoglobin, Cardiovascular, and Renal Outcomes in a Pharmacist-Managed Clinic. Annals of Pharmacotherapy, 38, 771-775. https://doi.org/10.1345/aph.1D359

[10] Gerber, R.A., Liu, G. and McCombs, J.S. (1998) Impact of Pharmacist Consultations Provided to Patients with Diabetes on Healthcare Costs in a Health Maintenance 
Organization. American Journal of Managed Care, 4, 991-1000.

[11] Cranor, C.W., Bunting, B.A., Christensen, D.B. (2003) The Asheville Project: Long-Term Clinical and Economic Outcomes of a Community Pharmacy Diabetes care Program. Journal of the American Pharmaceutical Association, 43, 173-184. https://doi.org/10.1331/108658003321480713

[12] Powell, M.F., Burkhart, V.D. and Lamy, P.P. (1979) Diabetic Patient Compliance as a Function of Patient Counseling. Annals of Pharmacotherapy, 13, 506-511. https://doi.org/10.1177/106002807901300904

[13] Odegard, P.S., Goo, A., Hummel, J., Williams, K.L., Gray, S.L. (2005) Caring for Poorly Controlled Diabetes Mellitus: A Randomized Pharmacist Intervention. Annals of Pharmacotherapy, 39, 433-440. https://doi.org/10.1345/aph.1E438

[14] Kiel, P.J. and McCord, A.D. (2005) Pharmacist impact on Clinical Outcomes in a Diabetes Disease Management Program via Collaborative Practice. Annals of Pharmacotherapy, 39, 1828-1832. https://doi.org/10.1345/aph.1G356 